\title{
Stringy instantons and quiver gauge theories
}

\author{
Bogdan Florea, ${ }^{a}$ Shamit Kachru, ${ }^{a b}$ John McGreevy ${ }^{a c}$ and Natalia Saulina ${ }^{d e}$ \\ ${ }^{a}$ Department of Physics and SLAC, Stanford University \\ Palo Alto, CA 94305-4060, U.S.A. \\ ${ }^{b}$ Kavli Institute for Theoretical Physics, University of California \\ Santa Barbara, CA 93106-4030, U.S.A. \\ ${ }^{c}$ Center for Theoretical Physics, Massachusetts Institute of Technology \\ Cambridge, MA 02139, U.S.A. \\ ${ }^{d}$ Department of Physics, Harvard University \\ Cambridge, MA 02138, U.S.A \\ ${ }^{e}$ Department of Physics, California Institute of Technology \\ Pasadena, CA 91125, U.S.A \\ E-mail: bflorea@slac.stanford.edu, skachru@stanford.edu, mcgreevy@mit.edu, \\ saulina@theory.caltech.edu
}

\begin{abstract}
We explore contributions to the $4 D$ effective superpotential which arise from Euclidean D3 branes ("instantons") that intersect space-filling D-branes. These effects can perturb the effective field theory on the space-filling branes by nontrivial operators composed of charged matter fields, changing the vacuum structure in a qualitative way in some examples. Our considerations are exemplified throughout by a careful study of a fractional brane configuration on a del Pezzo surface.
\end{abstract}

KEYWORDs: Flux compactifications, Superstring Vacua, D-branes. 


\section{Contents}

1. Introduction 且

2. The $d P_{1}$ quiver 3

3. Anomalous U(1)'s 5

3.1 Generalities

3.2 The axion charges in the cone over $d P_{1}$

3.2 .1 The surviving axions

3.2.2 The geometry of $B$

3.2.3 Pushing forward our branes 9

3.2.4 Axion charges 10

4. Strings between the instanton and the quiver 11

4.0.5 Twisted reduction 11

4.1 Ganor strings for a general class of bundles 12

5. Instanton effects 13

5.1 Which topologies can contribute? 13

5.2 BPS instantons 14

5.3 First example: The ADS instanton as a D-brane 15

5.4 Second example: A "stringy" deformation of the field theory superpotential 17

5.5 Contributions of D-string instantons 18

6. Vacuum structure: on the capture of the runaway quiver 19

A. Mixed anomalies 22

\section{Introduction}

Models of dynamical supersymmetry breaking are of great interest, both for the intricate dynamics they exhibit, and for their possible application to the hierarchy problem [1]. By definition, such models invoke non-perturbative effects in $4 \mathrm{~d}$ supersymmetric quantum field theory. In the context of string theory, where the $4 \mathrm{~d}$ low-energy supersymmetric theory arises via compactification, one expects that instanton effects in the field theories on spacefilling D-branes can be recast as (Euclidean) D-brane instanton effects [2]. Perhaps the simplest example is the theory on $N$ D3 branes; the Yang-Mills instanton in this theory is simply the $\mathrm{D}(-1)$ brane inside the $\mathrm{D} 3$ branes. 
More generally, other Euclidean D-branes which cannot be interpreted as instantons in non-Abelian gauge groups can also contribute to the superpotential, and hence may be important in the study of supersymmetry breaking. For instance in F-theory on an elliptic Calabi-Yau fourfold $X \rightarrow B$, Witten argued some time ago [3] that contributions to the superpotential for Kähler moduli arise from D3's which wrap divisors in $B$ that lift to vertical divisors of holomorphic Euler character $\chi=1$ in $X$. These effects, though non-perturbative in strength, can play a crucial role in low-energy physics. For example, they are useful in constructing controlled examples of moduli (meta)stabilization [4- 6 .

In cases where the instanton intersects other (space-filling) D-branes, the analysis of [3] does not immediately apply. It is then useful to find techniques to compute directly the superpotential in this situation. For instance, suppose that one has arranged for a nontrivial quiver field theory to arise at a singularity in $B$. That is, one has some nontrivial spectrum of D3, D5 and D7 branes wrapping various cycles in the singular geometry. Then Euclidean D3's which intersect the singularity, will in general have massless strings which stretch to the occupied nodes of the quiver (as was first discussed in some simple cases by Ganor [7]). These modes will couple to the quiver fields, and the integral over these collective coordinates will then pull down nontrivial operators involving fields in the quiver gauge theory. Schematically, if the Euclidean D3 wraps a cycle of volume $V$, and the quiver theory has charged fields $\phi_{i}$, one finds effects of the form

$$
\Delta W \sim \mathcal{O}\left(\phi_{i}\right) \operatorname{Exp}(-V) .
$$

We will see that the operators $\mathcal{O}$ generated in this way can have a qualitative effect on the spacetime physics.

The Kähler moduli chiral multiplets actually play a second, equally important role in these theories. The quiver gauge theories generically come with some number of anomalous U(1) factors. The anomalies are cancelled by the Green-Schwarz mechanism, whereby certain RR axions shift under the anomalous U(1) 8- 10]. These axions fill out (complex) scalar components of chiral multiplets in the supersymmetric effective theory; they are the partners of the Kähler moduli. Therefore, for Kähler moduli partnered with the charged axions, the associated instanton action is no longer gauge invariant! However, the relevant contributions (1.1) are generated in just such a way that the operator $\mathcal{O}$ carries opposite charge under all $\mathrm{U}(1)$ symmetries, yielding a gauge invariant contribution to $W$.

In this paper, we initiate the systematic study of such instanton effects. We focus on a particular example, the fractional brane in the Calabi-Yau cone over $d P_{1}$. In addition to providing an ideal expository example, this theory is of independent physical interest. It was conjectured to dynamically break supersymmetry in the pioneering works 11- 13. The thorough analysis in [14 proved that in a suitable decoupling limit, the theory has no stable vacuum. These authors also stressed that various possible perturbations of the superpotential could stabilize the model, as was further explored for a wide class of models in the recent work [15]. The decoupling limit of [14] involves taking the limit $M_{X} \rightarrow \infty$, where $M_{X}$ is the mass of the anomalous U(1) gauge bosons. In what follows, we will see that this is a subtle limit, since for any finite but large $M_{X}$ the behavior of the theory is qualitatively different. In particular, finite $M_{X}$ will arise in the compactified realizations 
of these models [16], assuming moduli can be stabilized. We will briefly discuss moduli stabilization in such examples at the end of this paper. A similar analysis can be carried out for many other quiver gauge theories; we leave this for the future.

The organization of the paper is as follows. In $\S 2$, we describe our main example, the quiver gauge theory arising from the Calabi-Yau cone over $d P_{1}$. Here we fix notation, describe the exceptional collection and various mathematical facts which we will use in our calculations, and discuss basic aspects of the fractional brane gauge theory. The field theory we find in $\S 2$ has two anomalous $\mathrm{U}(1)$ factors. In $\S 3$, we first briefly discuss why it is appropriate to keep these factors (and their associated D-terms) in the analysis of the vacuum structure of the compactified string theory. We then review the relevant GreenSchwarz mechanism, and compute the charges of the various axions under the anomalous U(1)'s. In $\S 4$, we compute the numbers of strings stretching from a Euclidean D3 to a D7, in terms of dimensions of appropriate cohomology groups. We then apply this to give concrete results for the spectrum of strings between a Euclidean D3 wrapping the $d P_{1}$ (with some general line bundle on its worldvolume), and the nodes of the quiver. In $\S 5$, we spell out the rules for instantons to contribute, and discuss two contributing instantons. One can be straightforwardly interpreted as the Affleck-Dine-Seiberg instanton in one of the quiver nodes, and contributes the expected field theory superpotential [17. The other is an instanton which does not lie entirely in any of the nodes of the quiver, and is in this sense a "stringy" effect which depends on the UV completion. It gives rise to a baryonic perturbation of the superpotential that qualitatively changes the low-energy physics. Finally, in $\S 6$, we discuss the vacuum structure of the resulting compactified quiver theory, including both the new instanton effect and the effect of the U(1) D-terms.

The effects we describe occur in any string compactification containing space-filling branes. For example, the contributions of D-instantons are also modified when the Standard Model lives on such D-branes, as in e.g. [18]. The possibility that $\mu$-terms are generated in this way has been pursued by [19]. Very recently [20], such effects were applied to neutrino masses in a similar context. While we were brooding over the selection rules implied by the R-anomaly, we also learned of the work [21, 22], which has some overlap with ours.

\section{The $d P_{1}$ quiver}

We will study branes in the complex cone over $d P_{1}$ (or equivalently $\mathbb{F}_{1}$ ). Since $d P_{1}$ has $h^{0,0}=h^{2,2}=1$ and $h^{1,1}=2$, a general brane is specified by four charges and the quiver will have four nodes. Denote the nontrivial two-cycles in $\mathbb{F}_{1}$ (the fiber and the base, viewing $\mathbb{F}_{1}$ as a $\mathbb{P}^{1}$ bundle over $\mathbb{P}^{1}$ ) as $f$ and $C_{0}$. Then a useful basis of branes is given by:

$$
\left\{\mathcal{L}_{1}, \mathcal{L}_{2}, \mathcal{L}_{3}, \mathcal{L}_{4}\right\}=\left\{\mathcal{O}_{\mathbb{F}_{1}}, \mathcal{O}_{\mathbb{F}_{1}}\left(C_{0}+f\right), \overline{\mathcal{O}_{\mathbb{F}_{1}}(f)}, \overline{\mathcal{O}_{\mathbb{F}_{1}}\left(C_{0}\right)}\right\}
$$

This quiver has one non-anomalous fractional brane, given by the combination

$$
[D 5]=\mathcal{L}_{1}+2 \mathcal{L}_{2}+3 \mathcal{L}_{3} .
$$

In addition, one can take any multiple $M \geq 1$ of this representation. The quiver summarizing the field content for general $M$ is given below. We will focus on the case $M=1$ for the most part. 


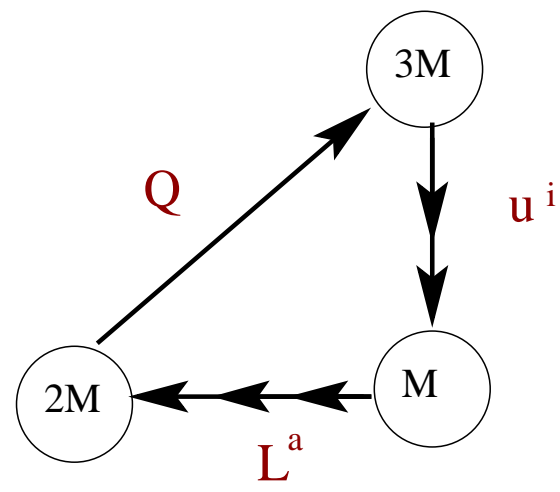

Figure 1: The quiver for the nonanomalous fractional brane wrapping $d P_{1}$.

The theory on $M$ fractional branes is characterized by gauge group $\mathrm{U}(3 M) \times \mathrm{U}(2 M) \times$ $\mathrm{U}(M)$. We will denote the corresponding $\mathrm{U}(1)$ factors as $\mathrm{U}(1)_{3}, \mathrm{U}(1)_{2}$ and $\mathrm{U}(1)_{1}$. The sum of the three U(1)'s decouples (all fields are neutral), while the two remaining combinations are in fact anomalous.

In an approximation where one discards the anomalous U(1)'s and their D-terms, the dynamics of the field theory with field content as in the quiver above and tree-level superpotential

$$
W_{\text {tree }}=\lambda Q \epsilon_{i j} u^{i} L^{j}
$$

(which preserves a global $\mathrm{SU}(2)_{F}$ flavor symmetry) was analyzed in detail in [14]. For convenience, we reproduce here the table from [14] summarizing the gauge and global symmetry representations of the various fields:

$\begin{array}{lcccccc} & \mathrm{U}(3 M) & \mathrm{U}(2 M) & \mathrm{U}(M) & {\left[\mathrm{SU}(2)_{F}\right.} & \mathrm{U}(1)_{F} & \left.\mathrm{U}(1)_{R}\right] \\ Q & \mathbf{3 M} & \overline{\mathbf{2 M}} & \mathbf{1} & \mathbf{1} & 1 & -1 \\ u & \overline{\mathbf{3 M}} & \mathbf{1} & \mathbf{M} & \mathbf{2} & -1 & 0 \\ L & \mathbf{1} & \mathbf{2 M} & \overline{\mathbf{M}} & \mathbf{2} & 0 & 3 \\ L_{3} & \mathbf{1} & \mathbf{2 M} & \overline{\mathbf{M}} & \mathbf{1} & -3 & -1\end{array}$

where the groups in the brackets are global symmetries of the theory.

The $\mathrm{U}(1)_{R}$ symmetry has triangle anomalies with the $\mathrm{U}(1)_{B}$ baryonic gauge symmetries

$$
\mathcal{A}\left(\mathrm{U}(1)_{R} \mathrm{U}(1)_{a} \mathrm{U}(1)_{b}\right)=-2 r_{a} r_{b} .
$$

Here $r_{a}=(1,2,-3)$ is the rank of the a-th node of the quiver. When the quiver gauge theory is realized in terms of $\mathrm{D}$-branes in the complex cone over $\mathbb{F}_{1}$, the R-anomaly can be cured ${ }^{1}$ by the coupling of the gauge fields to the $\mathrm{RR} 2$-form in $\mathbb{R}^{4}$ as shown in the appendix.

\footnotetext{
${ }^{1}$ In section 3 we consider compact model which involves an orientifold of IIB string theory and projects RR 2-form in $M^{4}$ out of the massless fields. Therefore, in the compact model the R-symmetry is broken. It must also be broken perturbatively in the compact model since such isometry would imply reduced holonomy. Note that this is particularly important in the $M>1$ case where 15 . has shown that no gauge invariant operator has the right R-symmetry to appear in the superpotential.
} 
Consider the $M=1$ case in the limit $\Lambda_{3} \gg \Lambda_{2}$, so that the $\mathrm{SU}(2)$ gauge factor can be treated as a global symmetry in the leading approximation. The SU(3) factor effectively has $N_{f}=2=N_{c}-1$, and so its dynamics generate a non-perturbative correction to (2.3) - the Affleck-Dine-Seiberg superpotential [17]:

$$
W_{\text {quantum }}=W_{\text {tree }}+\frac{\Lambda_{3}^{7}}{\operatorname{det}(Q \cdot u)} .
$$

The theory has no supersymmetric vacua, in keeping with the arguments of [11-13]. However, it has no stable vacuum at all [14].

We will see that the compactified string theory modifies the story in two interesting ways. Firstly, when the string scale is finite, the anomalous $\mathrm{U}(1)_{B} \mathrm{~s}$ do not decouple, and their anomalies are cancelled via the Green-Schwarz mechanism. Secondly, the quantum superpotential (2.6) can receive further contributions from "stringy" effects, that modify the story in an interesting way.

\section{Anomalous U(1)'s}

\subsection{Generalities}

In this subsection we briefly review the effective field theory of anomalous U(1)'s 8,23 , 24, 10. In the next, we compute the axion charges required to cancel anomalies in our theory. In many ways, the relevant Green-Schwarz mechanism is a fancy string theory realization of a simple field theory phenomenon, which often occurs when a gauge symmetry is spontaneously broken at a high scale.

In superspace, there are two terms that are important for anomaly inflow. Consider a model with a chiral multiplet $S$, an abelian vector multiplet $V_{X}$, and an $\mathrm{SU}(N)$ gauge group with which the $\mathrm{U}(1)$ has a mixed $\left(\mathrm{U}(1) \mathrm{SU}(N)^{2}\right)$ anomaly. The FI term and the mass for the $\mathrm{U}(1)$ gauge boson both arise from the gauge-invariant term

$$
L \supset \int d^{4} \theta K\left(S+S^{\dagger}+\delta V_{X}\right)=\left.V_{X}\right|_{\theta^{4}} K^{\prime}(s+\bar{s})+\frac{1}{2} K^{\prime \prime}(s+\bar{s})\left(\partial \phi+A_{X}\right)^{2}+\ldots,
$$

where $\phi=\operatorname{Im} s$, with $s$ being the lowest component of $S$. In string models where $\mathrm{U}(1)_{X}$ is an open-string gauge symmetry, $\phi$ is a mode of a closed string RR field. Under a $\mathrm{U}(1)_{X}$ gauge transformation, $A \mapsto A+d \lambda, \phi \mapsto \phi-\lambda$. Note that the first term on the r.h.s. of (3.1) can be interpreted as a (dynamical) FI term, while the second term is a field-dependent gauge boson mass $M_{X}$. In type II string models, this mass is moduli-dependent and can satisfy $M_{X} \ll M_{s}$ 10.

The other important term for anomaly inflow (which arises from one-loop gaugecoupling renormalization in the high energy theory) is

$$
L \supset \int d^{2} \theta S \operatorname{tr} W_{\alpha} W^{\alpha},
$$

the contribution of $S$ to the holomorphic gauge coupling of the nonabelian gauge group, whose gaugino superfield is $W^{\alpha}$. The shift of $\phi$ under the anomaly then cancels the anomalous variation of the action in an $\mathrm{SU}(N)$ instanton background. 
Now, one can ask, "how should $K$ depend on $S$ ?" As a simple field-theoretic example, if the axion arises as the phase of a chiral superfield $X$ that gets a high-scale vev, then the FI term comes from the ordinary canonical gauge invariant kinetic term for $X$ :

$$
\int d^{4} \theta X^{\dagger} e^{2 \delta_{X} V} X
$$

In this case, $\langle X\rangle=M e^{S}$ and so $K \sim e^{S+S^{\dagger}+2 \delta_{X} V}$. In string theory, the relevant Kähler potentials are more complicated, but the general structure remains as in (3.1).

Note that the massive U(1)'s, for any finite $M_{X}$, should not simply be thrown away. Their presence can qualitatively alter the vacuum structure. One can see this in two ways:

(i) Suppose the $U(1)_{X}$ gauge boson is relatively light compared to the cutoff $\Lambda_{U V}$ of the effective field theory, $M_{X} \ll \Lambda_{U V}$. Then, one should include the gauge supermultiplet in the low-energy effective theory, and impose the related D-term conditions. These change the potential and alter the vacuum structure. In a theory with chiral multiplets $\phi_{i}$ of charges $q_{i}$, the full potential will take the form

$$
V=\sum_{i}\left|\frac{\partial W}{\partial \phi_{i}}\right|^{2}-\frac{1}{2 g_{X}^{2}} D_{X}^{2}-\frac{1}{g_{X}^{2}} D_{X}\left(\sum_{i} q_{i}\left|\phi_{i}\right|^{2}-\xi\right)
$$

with $\xi$ the FI term.

(ii) Suppose instead that one is interested in constructing an effective theory integrating out the $\mathrm{U}(1), \Lambda_{U V} \leq M_{X}$. Integrating out the massive $\mathrm{U}(1)$ gauge boson generates a correction to the Kähler potential for the chiral multiplets 25]

$$
\delta K=-\frac{g_{X}^{2}}{M_{X}^{2}} q_{i} q_{j} \phi^{\dagger i} \phi_{i} \phi^{\dagger j} \phi_{j}
$$

This correction is of dimension greater than four, so one might feel that it cannot influence the vacuum structure. However, one can see that at a stationary point of the full potential $V(3.3)$, gauge invariance implies that

$$
\left\langle D_{X}\right\rangle=-\frac{g_{X}^{2}}{M_{X}^{2}} \sum_{i} q_{i}\left|\left\langle F_{i}\right\rangle\right|^{2}
$$

where

$$
M_{X}^{2}=g_{X}^{2} \sum_{i} q_{i}\left|\phi_{i}\right|^{2}
$$

In the theory with $\mathrm{U}(1)_{X}$ integrated out, the correction to the effective potential which arises from plugging nonzero $F_{i}$ into (3.4), then precisely reproduces the effect of the Dterms from the massive $\mathrm{U}(1)$ (at least as far as scalar soft masses are concerned).

One must be careful when using this logic. If $M_{X}$ exceeds some other threshold where new states are produced (notably $M_{s}$ in a generic string compactification), other corrections to $K$ will enter at the same order and should not be ignored. Then, there is no consistent effective theory which only includes the quartic correction (3.4) to the canonical Kähler potential. So for this formalism to be strictly useful, one should work in a regime where e.g. $M_{X} \ll M_{s}$. $M_{X}$ should be the first threshold where new physics is encountered. 


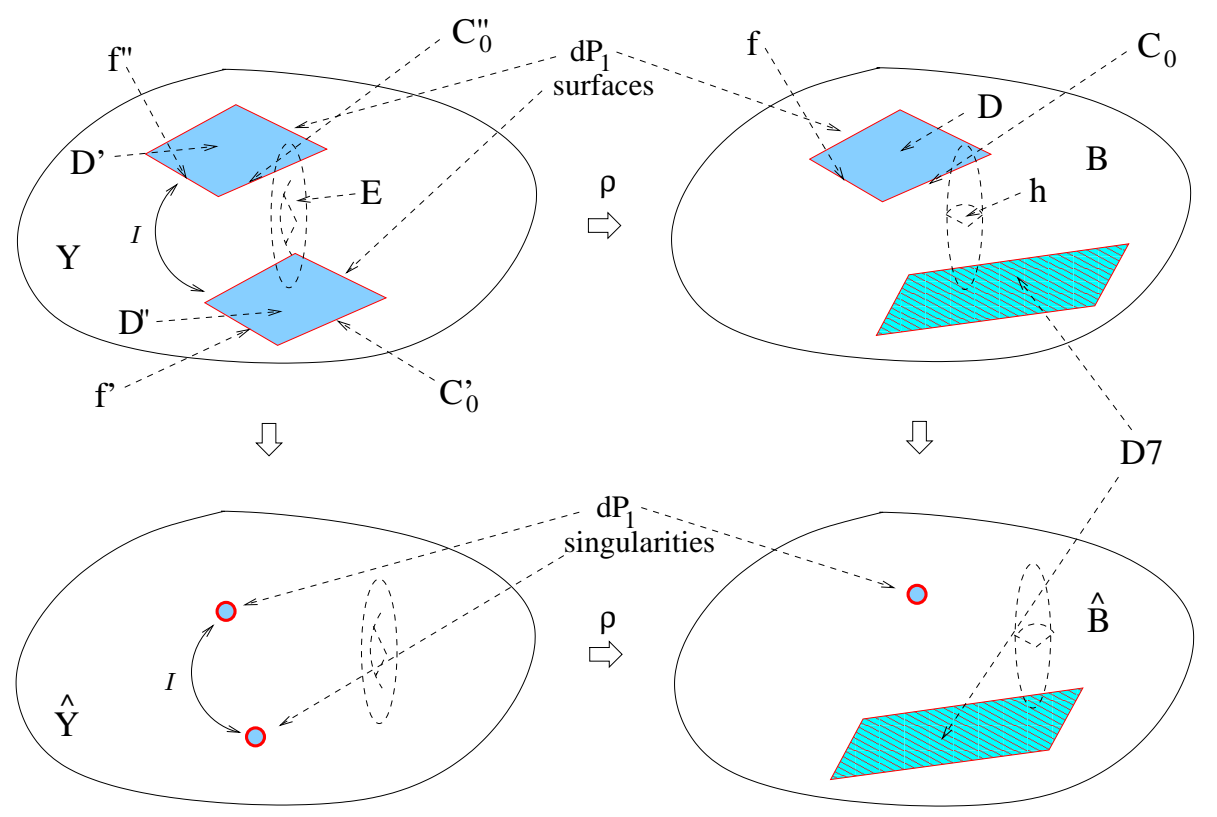

Figure 2: The Calabi-Yau singularity.

\subsection{The axion charges in the cone over $d P_{1}$}

To compute the anomaly-cancelling axion charges in our theory, it is useful to work in the context of a concrete compactification of the cone. We will use a construction modeled on the examples of [16].

We start with the projective threefold

$$
B \equiv \mathbb{P}\left(\mathcal{O}_{\mathbb{F}_{1}} \oplus \mathcal{O}_{\mathbb{F}_{1}}\left(\mathcal{K}_{\mathbb{F}_{1}}\right)\right)
$$

The negative section of $B$ is a contractible surface $D$ isomorphic to a del Pezzo $d P_{1}$. Consider now a Calabi-Yau hypersurface $Y$ given by a quadric in the toric fourfold $Z=$ $\mathbb{P}\left(\mathcal{O}_{B} \oplus \mathcal{O}_{B}\left(\mathcal{K}_{B}\right)\right) . Y$ is a torus fibration over $d P_{1}$ with two sections and has $h^{1,1}(Y)=$ $4, h^{2,1}(Y)=148$. There exists a $\mathbb{Z}_{2}$ involution $\mathcal{I}: Y \rightarrow Y$ such that the quotient $Y / \mathcal{I}$ is isomorphic to $B$ and the inverse image of $D$ under the map $\rho: Y \rightarrow B$ consists of a pair of surfaces $D^{\prime}, D^{\prime \prime}$ isomorphic to $d P_{1}$ which are contractible inside $Y$. The resulting singularities admit first order deformations which are obstructed at second order.

By construction, the local neighborhood of $D$ in $B$ is Calabi-Yau, so we can use facts about the quiver gauge theory that were derived by studying singular Calabi-Yau threefolds. In a more general compactification of F-theory on a fourfold, the local neighborhood of the singularity in $B$ would not necessarily be Calabi-Yau, and the results could differ.

\subsubsection{The surviving axions}

Before describing the geometry in more detail, we pause here to explain some differences between these models and more commonly studied D3/D7 orientifolds. One important fact is that because the involution exchanges two copies of $d P_{1}$ (call them $D$ and $D^{\prime}$ ), it 
also exchanges the curves $C_{0}, f \subset D$ with $C_{0}^{\prime}, f^{\prime} \subset D^{\prime}$. In the theory with no orientifold quotient, one would have obtained (from this part of the geometry) the six RR axions ${ }^{2}$

$$
\begin{aligned}
\phi_{C_{0}} & =\int_{C_{0}} C_{2}, & \phi_{C_{0}^{\prime}} & =\int_{C_{0}^{\prime}} C_{2} \\
\phi_{f} & =\int_{f} C_{2}, & \phi_{f^{\prime}} & =\int_{f^{\prime}} C_{2} \\
\phi_{\mathbb{F}_{1}} & =\int_{\mathbb{F}_{1}} C_{4}, & \phi_{\mathbb{F}_{1}^{\prime}} & =\int_{\mathbb{F}_{1}^{\prime}} C_{4} .
\end{aligned}
$$

The orientifold involution acts by $\mathcal{I}$ composed with $\Omega(-1)^{F_{L}}$ where $\Omega$ is worldsheet parity and $F_{L}$ is left-moving fermion number. Normally (see e.g. p.6 of [26]), the action of $\Omega(-1)^{F_{L}}$ on $C_{2}, C_{4}$ is $-1,+1$, and one projects out the $C_{2}$ axions. However, here, by taking appropriate combinations from the two copies of the shrinking del Pezzo, one gets both $C_{2}$ and $C_{4}$ axions. Namely, the combinations

$$
\begin{aligned}
\tilde{\phi}_{C_{0}} & =\phi_{C_{0}}-\phi_{C_{0}^{\prime}} \\
\tilde{\phi}_{f} & =\phi_{f}-\phi_{f^{\prime}} \\
\tilde{\phi}_{\mathbb{F}_{1}} & =\phi_{\mathbb{F}_{1}}+\phi_{\mathbb{F}_{1}^{\prime}}
\end{aligned}
$$

all survive the orientifold projection. Henceforth, to simplify life, we will abuse notation and call these surviving combinations $\phi_{C_{0}}, \phi_{f}$ and $\phi_{\mathbb{F}_{1}}$.

\subsubsection{The geometry of $B$}

$B$ can be described torically (or in the language of the gauged linear sigma model (GLSM) [27]) as follows. There are six chiral multiplets in the GLSM, charged under three $\mathrm{U}(1)$ gauge symmetries:

$$
\begin{array}{cccccc}
D_{1} & D_{2} & D_{3} & D_{4} & D_{5} & D_{6} \\
1 & 0 & 1 & 0 & -2 & 0 \\
0 & 1 & -1 & 1 & -1 & 0 \\
0 & 0 & 0 & 0 & 1 & 1
\end{array} .
$$

In terms of the geometry of the $d P_{1}$ described earlier, one should think of $D_{2}\left(D_{3}\right)$ as the $\mathbb{P}^{1}$-fibration over $f\left(C_{0}\right) . D_{5}$ is the $\mathbb{F}_{1}$ base.

It follows from the toric data that there are three linear equivalences:

$$
D_{2}=D_{4}, \quad D_{6}=D_{5}+2 D_{3}+3 D_{2}, \quad D_{1}=D_{2}+D_{3}
$$

The nonzero triple intersections are

$$
D_{5}^{3}=8, \quad D_{5} D_{2} D_{3}=1, \quad D_{5}^{2} D_{2}=-2, \quad D_{5}^{2} D_{3}=D_{5} D_{3}^{2}=-1 .
$$

\footnotetext{
${ }^{2}$ We work in the normalization that RR forms shift by integral closed forms under global gauge transformations.
} 
A consequence of the fact that a neighborhood of the del Pezzo is locally CY is the relation

$$
D_{5}^{2}=-2 D_{3} D_{5}-3 D_{2} D_{5} .
$$

It says that the self-intersection of the base is its own canonical curve.

Note from (3.11) that the geometry $B$ has an SU(2) symmetry under which the coordinates $D_{2}, D_{4}$ transform as a doublet. Any particular representative of the linear equivalence class of $D_{2}$ breaks this symmetry. This symmetry is the geometric origin of the $\mathrm{SU}(2)_{F}$ flavor symmetry of the field theory described in $\S 2$.

In order to compute the axion charges, we need to evaluate the topological terms in the brane worldvolume action. Recall that for a brane characterized by some sheaf $V_{\alpha}$ on $B$, these couplings are summarized by (in the units $2 \pi \alpha^{\prime}=1$ )

$$
\frac{i}{2} \int_{B} \sum_{p \text { even }} C^{(p)} \wedge \sqrt{\hat{A}(B)} \wedge \operatorname{ch} V_{\alpha} \wedge \operatorname{tr}_{\alpha} F \wedge F
$$

Therefore, we will need the Chern classes of $B$ and the bundles characterizing the quiver nodes.

The total Chern class of $B$ is

$$
c(B)=\prod_{i=1}^{6}\left(1+D_{i}\right)=\left(1+D_{2}+D_{3}\right)\left(1+D_{2}\right)^{2}\left(1+D_{5}\right)\left(1+D_{5}+2 D_{3}+3 D_{2}\right)\left(1+D_{5}\right) .
$$

It follows that the Todd class and A-roof genus of $B$ are

$$
\begin{aligned}
\operatorname{Td}(B) & =1+\frac{c_{1}}{2}+\frac{c_{1}^{2}+c_{2}}{12}+\ldots=1+D_{5}+2 D_{3}+3 D_{2}+\ldots \\
\hat{A}(B) & =e^{-\frac{c_{1}(B)}{2}} \operatorname{Td}(B) .
\end{aligned}
$$

\subsubsection{Pushing forward our branes}

In order to compute the Chern-Simons terms in the worldvolume Lagrangians, we will pull back the quiver branes to bundles on $B$. To do this we apply the Grothendieck-RiemannRoch $^{\mathrm{TM}}$ formula to the inclusion map $i: D_{5} \hookrightarrow B$. Applied to $\mathcal{L}_{1}=\mathcal{O}_{\mathbb{F}_{1}}$, this says

$$
i_{\star}\left(\operatorname{ch} \mathcal{L}_{1} \operatorname{Td}\left(D_{5}\right)\right)=\operatorname{ch}\left(i_{\star} \mathcal{L}_{1}\right) \operatorname{Td}(B) .
$$

Using $\operatorname{Td}\left(D_{5}\right)=1+C_{0}+\frac{3}{2} f+\left[\mathrm{pt}_{\mathbb{F}_{1}}\right], \operatorname{ch}\left(\mathcal{L}_{1}\right)=1$, and

$$
i_{\star}\left(\operatorname{ch} \mathcal{L}_{1} \operatorname{Td}\left(D_{5}\right)\right)=D_{5}+D_{5} D_{3}+\frac{3}{2} D_{5} D_{2}+[\mathrm{pt}]
$$

we get

$$
\operatorname{ch}\left(i_{\star} \mathcal{L}_{1}\right)=D_{5}-D_{5}^{2}-D_{3} D_{5}-\frac{3}{2} D_{2} D_{5}+\ldots=D_{5}+D_{3} D_{5}+\frac{3}{2} D_{2} D_{5}+\ldots
$$

Throughout, we are dropping "6-form" terms in the computation that would only determine the charges of the 10d RR axion $C_{0}-$ it follows from [10] that $C_{0}$ does not shift in the relevant Green-Schwarz mechanism. 
Similarly, we find

$$
\begin{aligned}
\operatorname{ch}\left(i_{\star} \mathcal{L}_{2}\right) & =D_{5}-D_{5}^{2}-\frac{1}{2} D_{2} D_{5}+\ldots \\
& =D_{5}+2 D_{3} D_{5}+\frac{5}{2} D_{2} D_{5}+\ldots \\
\operatorname{ch}\left(i_{\star} \mathcal{L}_{3}\right) & =-D_{5}+D_{5}^{2}+D_{3} D_{5}+\frac{1}{2} D_{2} D_{5}+\ldots \\
& =-D_{5}-D_{3} D_{5}-\frac{5}{2} D_{2} D_{5}+\ldots
\end{aligned}
$$

\subsubsection{Axion charges}

Finally, we can identify the charges we should assign to various RR axions, to cancel the worldvolume anomalies. The worldvolume theory of a D-brane on $B$ with gauge sheaf $V_{\alpha}$ contains the coupling (3.13). Note that we are assuming that the gauge bundle factorizes between $B$ and the four noncompact directions. In keeping with our earlier definitions, we let

$$
\phi_{f}=\int_{B} C^{(2)} \wedge D_{5} \wedge D_{2}, \quad \phi_{C_{0}}=\int_{B} C^{(2)} \wedge D_{5} \wedge D_{3}, \quad \phi_{\mathbb{F}_{1}}=\int_{B} C^{(4)} \wedge D_{5}=\int_{\mathbb{F}_{1}} C^{(4)} ;
$$

we are using a notation where $\int_{B} D \wedge \eta=\int_{D} \eta(\forall \eta)$, so $D$ is a two-form.

The anomalous variation of the worldvolume fermion measure (which captures the mixed anomalies of $\mathrm{U}(1)_{1,2,3}$ with the $\mathrm{SU}(M), \mathrm{SU}(2 M)$ and $\mathrm{SU}(3 M)$ factors, respectively $)^{3}$ is

$$
\begin{aligned}
\delta \Gamma=\frac{i}{2}\left(-\operatorname{tr}_{1} F \wedge F\left[+0 \lambda_{1}-6 M \lambda_{2}+6 M \lambda_{3}\right]-\right. \\
\operatorname{tr}_{2} F \wedge F\left[+3 M \lambda_{1}+0 \lambda_{2}-3 M \lambda_{3}\right]- \\
\left.\operatorname{tr}_{3} F \wedge F\left[-2 M \lambda_{1}+2 M \lambda_{2}+0 \lambda_{3}\right]\right)
\end{aligned}
$$

where $\lambda_{1,2,3}$ are gauge parameters for $\mathrm{U}(1)_{1,2,3}$. We set this equal to minus the variation of

$$
\begin{aligned}
S_{\mathrm{CS}}=\frac{i}{2}\left(\operatorname{tr}_{1} F \wedge F\left[\phi_{\mathbb{F}_{1}}+0 \phi_{f}+0 \phi_{C_{0}}\right]+\right. \\
\operatorname{tr}_{2} F \wedge F\left[\phi_{\mathbb{F}_{1}}+\phi_{f}+\phi_{C_{0}}\right]+ \\
\left.\operatorname{tr}_{3} F \wedge F\left[-\phi_{\mathbb{F}_{1}}-\phi_{f}+0 \phi_{C_{0}}\right]\right)
\end{aligned}
$$

(note that the $D_{5}^{2}$ term in the chern characters contributes through the relation (3.12)). We find, in multiples of $M$, the following charges

$\begin{array}{cccc} & \mathrm{U}(1)_{1} & \mathrm{U}(1)_{2} & \mathrm{U}(1)_{3} \\ e^{i \phi_{C_{0}}} & 1 & 2 & -3 \\ e^{i \phi_{f}} & 2 & 4 & -6 \\ e^{i \phi_{\mathbf{F}_{1}}} & 0 & -6 & 6 .\end{array}$

\footnotetext{
${ }^{3}$ The same choice of local counterterms will also cancel variations from mixed $\mathrm{U}(1)_{a} \mathrm{U}(1)_{b} \mathrm{U}(1)_{c}(a, b, c \in$ $\{1,2,3\})$ anomalies.
} 
A check on our algebra is the fact that no field transforms under the overall decoupled $\mathrm{U}(1)$, which has $\lambda_{1}=\lambda_{2}=\lambda_{3}$-i.e., the sum of the entries in each row vanishes. Note that the combination $2 \phi_{C_{0}}-\phi_{f}$ is neutral; $2 C_{0}-f$ is the cohomology class of the net D5 brane charge of the fractional brane.

\section{Strings between the instanton and the quiver}

To determine whether a Euclidean D3 contributes to the superpotential, we will need to know about the topology of the cycle it wraps in the Calabi-Yau, and we will need to determine its spectrum of "Ganor strings" - strings which stretch from the Euclidean brane to the (occupied) nodes of the quiver. Such strings act as collective coordinates of the instanton.

In our situation, both the Euclidean D3's and the quiver nodes wrap a surface $S \subset B$ (the $\mathbb{F}_{1}$ ), and carry different line bundles $\mathcal{L}_{A}, \mathcal{L}_{B}$ over $S$. The correct way to work out the spectrum is then to do a twisted reduction of the $D 3-D 7$ hypermultiplet on $S$.

\subsubsection{Twisted reduction}

Here we discuss the twisted reduction of the hypermultiplet of 3-7 strings, following a similar analysis for M5-brane fields in [3].

The normal bundle to a del Pezzo surface $S$ decomposes as $N \oplus T \mathbb{R}^{4}$, where $N$ is the normal bundle to $S$ in $B$. Because we are studying a situation where the neighborhood of the surface $S$ is Calabi-Yau, $N=K$, where $K$ is the canonical bundle of $S$. Following [3], we can identify the rotations of this normal bundle to the divisor with an R-symmetry of the field theory. This is because the superspace coordinates of the instanton arise from 3-3 strings; these transform as sections of the spin bundle derived from $N, S^{\prime} \equiv N_{1}^{1 / 2} \oplus N_{-1}^{-1 / 2}$, where the subscripts denote R-charges.

In the neighborhood of the del Pezzo singularity, the ten dimensional Lorentz group is broken to

$$
\mathrm{SO}(4)_{\mathbb{R}^{4}} \times \mathrm{SO}(4)_{d P_{1}} \times \mathrm{SO}(2)_{\perp} \subset \mathrm{SO}(10) .
$$

Let $S_{ \pm}^{\prime \prime}$ be the (trivial) chiral spin bundles on $\mathbb{R}^{4}$. The spin bundle ${ }^{4}$ on $d P_{1}$ decomposes into chiral parts as $S_{+} \oplus S_{-}$where

$$
\begin{aligned}
& S_{+}=K^{1 / 2} \oplus\left(K^{1 / 2} \otimes \Omega^{0,2}\right) \\
& S_{-}=K^{1 / 2} \otimes \Omega^{0,1} .
\end{aligned}
$$

Using the facts about $\#_{N D}=4$ systems (page 162 of [29]), we find that the fermions transform as sections of

$$
\left(S^{\prime} \otimes S_{+} \otimes \mathcal{L}_{A} \otimes \mathcal{L}_{B}^{\star}\right) \oplus\left(S^{\prime} \otimes S_{-} \otimes \mathcal{L}_{A}^{\star} \otimes \mathcal{L}_{B}\right)
$$

\footnotetext{
${ }^{4}$ Note that while $d P_{1}$ is not spin, implying that there is a Freed-Witten anomaly for branes wrapping it, it is $\operatorname{spin}^{\mathrm{c}}$. Both the D7-brane gauge bundle and the D3-brane gauge bundle are twisted by $K^{1 / 2}$ [28]. Since we will be interested in the dynamics of strings stretching between them, these factors cancel and this anomaly does not affect our analysis.
} 
Using $N=K$, this is

$$
\mathcal{L}_{A} \otimes \mathcal{L}_{B}^{\star} \otimes\left(K_{1} \oplus\left(K_{1} \otimes \Omega^{0,2}\right) \oplus \mathcal{O}_{-1} \oplus \Omega_{-1}^{0,2}\right) \oplus \mathcal{L}_{A}^{\star} \otimes \mathcal{L}_{B} \otimes\left(\left(K_{1} \otimes \Omega^{0,1}\right) \oplus \Omega_{-1}^{0,1}\right) .
$$

The dimensions of these summands, labelled by $4 \mathrm{~d}$ chirality and R-charge, are:

$$
\begin{aligned}
\left(h^{0,2}\left(\mathcal{L}_{A}^{\star} \otimes \mathcal{L}_{B}\right)_{1}+h^{0,0}\left(\mathcal{L}_{A}^{\star} \otimes \mathcal{L}_{B}\right)_{1}+h^{0,0}(\right. & \left.\left.\mathcal{L}_{A} \otimes \mathcal{L}_{B}^{\star}\right)_{-1}+h^{0,2}\left(\mathcal{L}_{A} \otimes \mathcal{L}_{B}^{\star}\right)_{-1}\right) \\
& +\left(h^{0,1}\left(\mathcal{L}_{A} \otimes \mathcal{L}_{B}^{\star}\right)_{1}+h^{0,1}\left(\mathcal{L}_{A}^{\star} \otimes \mathcal{L}_{B}\right)_{-1}\right) .
\end{aligned}
$$

In deriving (4.5) we have used Serre duality:

$$
\left(H^{0}\left(\Omega^{0, p} \otimes K \otimes L\right)\right)^{\star}=H^{0}\left(\Omega^{0,2-p} \otimes L^{\star}\right) .
$$

The bosons transform as sections of $\left(S_{+}^{\prime \prime} \otimes \mathcal{L}_{A} \otimes \mathcal{L}_{B}^{\star}\right) \oplus\left(S_{-}^{\prime \prime} \otimes \mathcal{L}_{A}^{\star} \otimes \mathcal{L}_{B}\right)$ (recall that a hypermultiplet contains an $\mathrm{SU}(2)$ doublet of complex bosons); the trivial spin bundles $S_{ \pm}^{\prime \prime}$ over $\mathbb{R}^{4}$ simply give the bosons multiplicity two.

In the following we have weighted the spectrum by signs in order to omit modes which can pair up in a manner consistent with gauge invariance and R-charge conservation. The end result is that the spectrum consists of:

- A net number of bosons given by

$$
n_{\text {bos }}\left(\mathcal{L}_{A}, \mathcal{L}_{B}\right)=2\left(h^{0}\left(S, \mathcal{L}_{A} \otimes \mathcal{L}_{B}^{*}\right)-h^{0}\left(S, \mathcal{L}_{B} \otimes \mathcal{L}_{A}^{*}\right)\right) .
$$

- A net number of fermions given by

$$
n_{\text {ferm }}\left(\mathcal{L}_{A}, \mathcal{L}_{B}\right)=\chi\left(\mathcal{L}_{A} \otimes \mathcal{L}_{B}^{*}\right)-\chi\left(\mathcal{L}_{A}^{*} \otimes \mathcal{L}_{B}\right)
$$

where

$$
\chi\left(\mathcal{L}_{A} \otimes \mathcal{L}_{B}^{*}\right)=\sum_{i=1}^{3}(-1)^{p} h^{p}\left(S, \mathcal{L}_{A} \otimes \mathcal{L}_{B}^{*}\right) .
$$

\subsection{Ganor strings for a general class of bundles}

Next we apply the results of the previous subsection to compute the spectra of Ganor strings for general instantons in the $d P_{1}$ example. These will be well summarized by extended quiver diagrams, where one includes a node for the instanton appended to the regular quiver, and adds arrows for the strings which stretch between the instanton and the quiver nodes. In general, one can then read off gauge invariant couplings between the instanton collective coordinates and the quiver fields, which will be relevant when evaluating the integral over the instanton modes (which is really an ordinary integral, not a functional integral).

The most general line bundle of interest for us here is $X_{a b}=\overline{\mathcal{O}_{D_{5}}\left(a C_{0}+b f\right)}$. The instantons multi-wrapping $D_{5}$ give (in the geometric regime) corrections which are exponentially smaller than the ones we compute; it would be necessary to include them to obtain any exact formula for the full superpotential, but we leave this for future research. The D3-instantons wrapping divisor in a class $\left[D_{5}+c D_{3}+d D_{2}\right]$ for non-zero $c$ and/or $d$ 
intersect "divisor at infinity" $D_{6}$. Therefore, such instantons have more zero modes and are likely to vanish; the detailed analysis of their contribution is model-dependent. We now compute the cohomology groups using e.g. [30],

$$
h^{0}\left(d P_{1}, \mathcal{O}\left(n C_{0}+m f\right)\right)=\#\left\{(x, y) \in \mathbb{Z}^{2} \mid x \geq-m, y \geq 0,-x-y \geq 0,-y \geq-n\right\}
$$

and Euler number of the bundle on $D_{5}$ using the GRR formula

$$
\chi(X)=\int_{D_{5}} \operatorname{ch}(X) \wedge \operatorname{Td}\left(D_{5}\right) .
$$

We find that the number of fermionic zero modes between $X_{a b}$ and $\mathcal{L}_{1,2,3}$ is

$$
n_{\text {ferm }}\left(X_{a b}, \mathcal{L}_{1,2,3}\right)=(a+2 b,-3+a+2 b, 2-a-2 b) .
$$

Note that the answer depends only on $a+2 b$ which we from now on set equal to 2 so that the instantons have $\mathrm{U}(1)_{B}$ charges $(2,-2,0)$. This choice is motivated by the fact that operators of greatest interest need those charges cancelled.

The number of bosonic zero modes depends on $b$. There are no bosonic zero modes for $b>1$ and $b \leq-1$, meanwhile for $b=1$ we find

$$
n_{\text {bos }}\left(X_{01}, \mathcal{L}_{1}\right)=4, \quad n_{\text {bos }}\left(X_{01}, \mathcal{L}_{2}\right)=-2, \quad n_{\text {bos }}\left(X_{01}, \mathcal{L}_{3}\right)=0 .
$$

\section{Instanton effects}

Equipped with the results of $\S 4$, we can now try to classify instantons by considering arbitrary line bundles on $S$ and integrating out their Ganor strings. The contribution of each such instanton is proportional to an exponential of $\mathrm{RR}$ axions, and therefore carries a definite set of $U(1)_{B}$ gauge charges by the results of $\S 3.2$. One check on our results should be that each non-vanishing contribution enjoys a cancellation of $\mathrm{U}(1)_{B}$ charges between the instanton action and the field theory operator arising from the integral over Ganor strings.

\subsection{Which topologies can contribute?}

Before we consider explicit examples, we would like to discuss which instantons can contribute to the superpotential. Let us first recall the constraints on topology of the instantons given in [3]. Using the duality between F-theory and M-theory, each Euclidean D3 instanton can be related to an M5 brane instanton in the F-theory fourfold $X$, wrapping some divisor $D_{M}$. In [3], Witten argued that, in the absence of the background fluxes, the necessary condition for D3-instantons to contribute is $\chi\left(D_{M}\right)=1$, where

$$
\chi\left(D_{M}\right)=\sum_{p=1}^{3}(-1)^{p} h^{0, p}\left(D_{M}\right) .
$$

$\chi\left(D_{M}\right)=1$ is necessary but not sufficient for instanton to generate $\mathrm{W}$ since it counts 3-3 fermion zero modes with sign. The sufficient condition is, of course, to saturate all fermion zero modes. 
There are two kinds of zero modes of interest: those from 3-3 strings, and those from 3-7 strings. As shown in [31], in the presence of gauge flux $F$ on the world-volume of a D3brane, the Dirac operator acting on world-volume fermions is deformed (see eq. 69 of [31]). This allows a possibility of lifting some or (in the lucky case) all of the non-universal 3-3 fermion zero modes, i.e. modes which come, in the M-theory language, from $h^{0, p}\left(D_{M}\right)$ for $p>1$. (As mentioned in [31] one might also find extra zero modes from $h^{0,0}\left(K, D_{5}\right)$; for the case at hand with $D_{5}$ a del Pezzo, this group vanishes).

More generally, one can worry that because the Euclidean D3 brane is wrapping a cycle disjoint from the O-planes in a Calabi-Yau threefold, it breaks (locally) half of the $N=2$ supersymmetry, and will have 4 fermion zero modes from the broken supercharges. While in the full theory the supersymmetry is really broken to $N=1$, the local brane physics will then see extra zero modes. However, there is also another mechanism of lifting (in some cases) non-universal 3-3 fermion zero modes, which is due to background three-form fluxes 32-34. We believe this would reduce the number of 3-3 zero modes to the expected 2 in many cases of interest; several groups are now investigating the precise circumstances under which this occurs.

When the D3-instanton intersects space-filling branes, the condition for the instanton to contribute is modified since 3-7 and 7-3 zero modes, discussed in $\S 4$, should be saturated as well. In general, integrating out these 3-7 and 7-3 zero modes leads to some operator $\mathcal{O}_{D}$ in the quiver gauge theory to arise as a prefactor of exponent of instanton action. The subscript is a reminder that the precise operator is determined by the integral over the instanton collective coordinates; it is a function of the instanton.

\subsection{BPS instantons}

One could worry that a semiclassical description involving branes wrapping a weakly curved space might break down near the quiver locus in Kähler moduli space where D7 branes and anti-D7 branes are simultaneously BPS. However, we are computing corrections to the superpotential, which is a holomorphic function of chiral superfields. Since the partners of Kähler moduli are axions, it is natural to expect that all of the dependence of the superpotential on the Kähler moduli appears in exponentials. We will make this assumption.

At the quiver locus in Kähler moduli space, where the quiver gauge theory exists, the central charges of the four sheaves on $\mathbb{F}_{1}$

$$
\mathcal{O}_{\mathbb{F}_{1}}, \quad \mathcal{O}_{\mathbb{F}_{1}}(h), \quad \overline{\mathcal{O}_{\mathbb{F}_{1}}(f)}, \quad \overline{\mathcal{O}_{\mathbb{F}_{1}}\left(C_{0}\right)}
$$

are aligned, i.e., have the same phase. The central charge $Z\left(X_{a b}\right)$ of D3-instanton with bundle $X_{a b}$ can be expressed as linear combination:

$$
\begin{aligned}
Z\left(X_{a b}\right)= & \frac{1}{2}\left(a^{2}-2 a b+a+2 b-2\right) Z\left(\mathcal{O}_{\mathbb{F}_{1}}\right)+\frac{1}{2}\left(a^{2}-a-2 a b\right) Z\left(\mathcal{O}_{\mathbb{F}_{1}}(h)\right) \\
& +\frac{1}{2}\left(a^{2}-a+2 b-2 a b\right) Z\left(\overline{\mathcal{O}_{\mathbb{F}_{1}}(f)}\right)+\frac{1}{2}\left(a^{2}-2 a b+a\right) Z\left(\overline{\mathcal{O}_{\mathbb{F}_{1}}\left(C_{0}\right)}\right) .
\end{aligned}
$$

For $a+2 b=2$ all coefficients in the linear combination (5.2) are non-negative. Therefore, all of the instantons $X_{a b}$ are aligned with nodes of the quiver. This ensures that such 


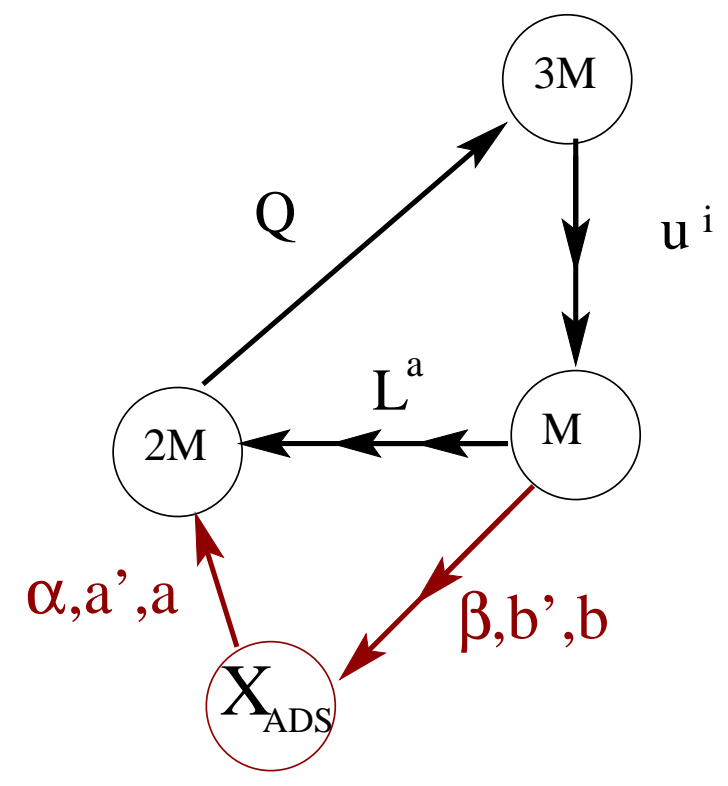

Figure 3: The extended quiver for the SU(3) instanton $X_{\mathrm{ADS}} \equiv X_{01}$.

instantons are BPS and 3-3 strings have two universal fermion zero modes $\theta^{\alpha}$ coming from breaking half of $\mathcal{N}=1$ supersymmetry in $\mathbb{R}^{4}$.

Please note that in the absence of gauge flux on D3-instanton, there are also two nonuniversal zero modes $2 h^{(0,2)}\left(K, D_{5}\right)=2$. These are lifted by the world-volume gauge flux $[F]=a C_{0}+b f$ as follows from the analysis of deformed world-volume Dirac operator [31].

Finally, there are 3-7 and 7-3 zero modes discussed in $\S 4$ which we integrate out in the next section.

\subsection{First example: The ADS instanton as a D-brane}

Consider our quiver for $M=1$. The SU(3) node has two flavors of quarks, and should generate a superpotential via the Affleck-Dine-Seiberg instanton effect [17]. (It is important that precisely for $N_{f}=N_{c}-1$, the dynamical superpotential in SUSY QCD is actually an instanton effect, and not a result of other strong dynamics as it is for $N_{f} \leq N_{c}-2$ ). A D3 brane wrapping $\mathbb{F}_{1}$ with the same bundle as $\mathcal{L}_{3}$ has precisely the correct action to be interpreted as the gauge theory instanton. This is the special case of 4.11) with $a=0, b=1$. Can we compute this contribution to the superpotential by quantizing its Ganor strings? (The reader is advised to see also 35 for a telegraphic discussion of a closely-related question.)

The appropriate extended quiver diagram, including a node for the instanton and the Ganor strings, appears below:

This case is special in that $X_{01} \otimes \mathcal{L}_{\alpha}$ have holomorphic sections; the analysis of the previous section then implies that there are bosonic zeromodes $a, b_{j}, a^{\prime}, b_{j}^{\prime}$, in addition to the fermions $\alpha, \beta_{j}$. The gauge charges are as indicated by the arrows in 3. A gauge invariant coupling between the quiver fields and the collective coordinates, which should be generated at the 
disc level, is

$$
\tilde{L}=\alpha Q \cdot u^{i} M_{i}^{j} \beta_{j}+a Q \cdot u^{i} N_{i}^{j} b_{j}+a^{\prime} Q \cdot u^{i} N_{i}^{\prime} j b_{j}^{\prime}
$$

where $M, N, N^{\prime}$ are some flavor matrices.

The contribution of the instanton $X_{01}$ is proportional to

$$
e^{-S_{\text {instanton }}}=e^{-S_{1}+\theta^{\alpha} \psi_{\alpha}+\tilde{L}}
$$

where

$$
S_{1}:=t_{\mathbb{F}_{1}}+t_{f}, \quad t_{\mathbb{F}_{1}}=\int_{B}\left(\frac{1}{4 \pi g_{s}} J^{2}+i C_{4}\right) D_{5}, \quad t_{f}=\int_{B}\left(\frac{1}{2 \pi g_{s}} J+i C_{2}\right) D_{2} D_{5} .
$$

$\theta^{\alpha}$ are two fermion zeromodes of 3-3 strings and $\psi_{\alpha}$ are fermion superpartners of $S_{1}$. Volumes are measured in string units $\alpha^{\prime}=\frac{1}{2 \pi}$ and as in section $3 \mathrm{RR}$ fields are normalized to have integral periodicity.

If we parametrize Kähler form $J$ as

$$
J=r_{5} D_{5}+r_{2} D_{2}+r_{3} D_{3}
$$

we find

$$
\operatorname{Re}\left(S_{1}\right)=\frac{1}{2}\left(8 r_{5}^{2}-r_{3}^{2}-2 r_{3} r_{5}-4 r_{2} r_{5}+2 r_{2} r_{3}\right)+r_{3}-2 r_{5}
$$

To find the superpotential we compute two-point correlator of fermions $\psi_{\alpha}$ in the instanton background:

$$
\left\langle\psi_{1}^{\dagger} \psi_{2}^{\dagger}\right\rangle_{\text {instanton }}=G_{\psi_{1}} G_{\psi_{2}} \partial_{S_{1}} \partial_{S_{1}} W
$$

where $G_{\psi}=\left\langle\psi^{\dagger} \psi\right\rangle$. Integral over 3-3 fermion zero modes pulls down $\psi_{1} \psi_{2}$ and we are left with integral over the 3-7 zero modes $a, b, a^{\prime}, b^{\prime}, \alpha, \beta$ :

$$
\partial_{S_{1}} \partial_{S_{1}} W=e^{-S_{1}} \int d a d^{2} b d a^{\prime} d^{2} b^{\prime} d \alpha d^{2} \beta e^{\tilde{L}} \sim \frac{\operatorname{det} M \operatorname{det}_{f j}\left(Q^{f} \cdot u_{j}\right)}{\operatorname{det} N \operatorname{det} N^{\prime} \operatorname{det}_{f j}^{2}\left(Q^{f} \cdot u_{j}\right)}
$$

From (5.5) we immediately find the following contribution to the superpotential ${ }^{5}$

$$
W_{\mathrm{ADS}}=\frac{\Lambda^{7}}{Z} e^{-S_{1}}
$$

where $Z=\operatorname{det}_{f j}\left(Q^{f} \cdot u_{j}\right)$ and $\Lambda^{7}$ comes from path integral normalization.

Note that:

- The factor of two in the multiplicity of bosons (4.7) was crucial for obtaining the ADS result.

- The U(1) gauge charges of the instanton action (inferred from the axion couplings) nontrivially cancel with those of $\mathcal{O}$ to yield a gauge invariant operator.

- Other couplings besides (5.3) are conceivable. For example, the full disc amplitude could give $\tilde{L}=f(\alpha Q u \beta)$ where $f$ is some more general function than $f(x)=x$. The important point is that it can only depend on these combinations of fields, and changing variables in the $\alpha, \beta$ integrand by e.g. $\alpha^{\prime} \equiv \alpha Q \cdot u, \beta^{\prime} \equiv \beta$ gives the expression above, times some integral does not depend on the quiver fields. This remark applies in all the cases we will discuss.

\footnotetext{
${ }^{5} \Lambda \equiv \Lambda_{3}$.
} 


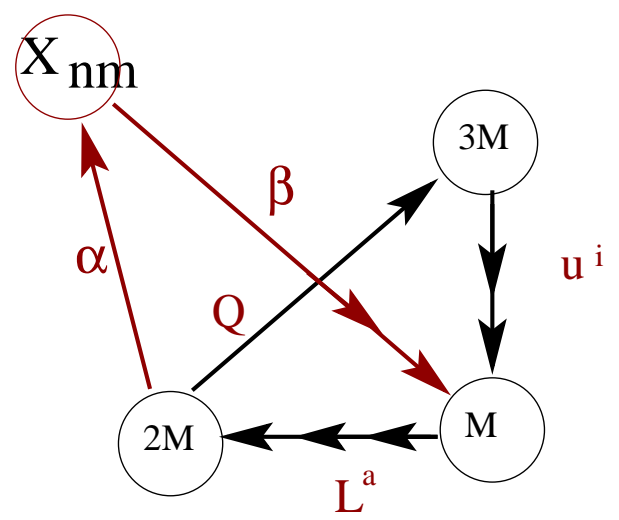

Figure 4: The extended quiver for the $X_{a b}$ instantons, when $2=a+2 b$ and $b>1$ or $b \leq-1$. $\alpha, \beta$ are fermionic.

\subsection{Second example: A "stringy" deformation of the field theory superpotential}

Now, consider the case $b>1$ or $b \leq-1$. The relevant extended quiver diagram appears below:

A gauge invariant coupling which is expected to arise from a disc amplitude is

$$
L=\alpha L^{a} M_{a j} \beta^{j}
$$

where $M$ is some flavor matrix which breaks the $\mathrm{SU}(2)_{F}$ and the $\mathrm{SU}(2)$ rotating the $\beta^{j}$ down to a diagonal subgroup. It is worth noting that the $\mathrm{SU}(2)$ which rotates the fermion zeromodes has the same origin as the $\mathrm{SU}(2)_{F}$. It arises from the action of the $\mathrm{SU}(2)$ isometry of the $d P_{1}$ on its cohomology. The two classes generating the $\beta^{j}$ arise from the $\mathrm{SU}(2)$ doublet of sections $z^{j}$ of $\mathcal{O}(1)$ over the sphere acted on by this $\mathrm{SU}(2)$. It is therefore not at all surprising to find that this geometric coupling preserves only one $\mathrm{SU}(2)_{F}$.

Integrating over the fermions $\alpha, \beta$ generates an operator of the form $\sum_{a} c_{a} \mathcal{O}_{a}$, where $\mathcal{O}_{a} \sim L^{b} L^{c} \epsilon_{a b c}$. What are the values of the coefficients $c_{a}$ ? In the field theory (2.4), $L_{1,2}$ form an $\mathrm{SU}(2)_{F}$ doublet while $L_{3}$ is an $\mathrm{SU}(2)_{F}$ singlet. Since the toric 3 -fold $B$ has $\mathrm{SU}(2)_{F}$ as a global symmetry, we have $c_{1}=c_{2}=0$. Including the dependence on Kähler moduli, the "stringy" instanton correction to $W$ has the form, ${ }^{67}$

$$
W_{\text {stringy }}=\frac{\Lambda^{7}}{M_{s}^{6}} V_{3} \sum_{b>1 \& b \leq-1} f(b) e^{-S_{1}+(b-1) S_{2}} .
$$

$S_{1}$ is defined in (5.4) and $S_{2}$ is given by

$$
S_{2}:=t_{f}-2 t_{C_{0}}, \quad t_{f}=\int_{B}\left(\frac{1}{2 \pi g_{s}} J+i C_{2}\right) D_{2} D_{5}, \quad t_{C_{0}}=\int_{B}\left(\frac{1}{2 \pi g_{s}} J+i C_{2}\right) D_{3} D_{5} .
$$

If we parametrize the Kähler form $J$ as before

$$
J=r_{5} D_{5}+r_{2} D_{2}+r_{3} D_{3}
$$

\footnotetext{
${ }^{6}$ This corrects statements made in $[36]$.

${ }^{7}$ The contribution of $X_{20}$ to the superpotential is zero because of unpaired bosonic zeromodes.
} 
we find

$$
\operatorname{Re}\left(S_{2}\right)=3 r_{3}-2 r_{2}
$$

We do not know the function $f(b)$, but we conjecture that it is such that $\sum_{b>1 \& b<-1} f(b)$ is convergent. $W_{\text {stringy }}$ is valid near the quiver locus $\left|\operatorname{Re}\left(S_{2}\right)\right| \ll 1$ so that $\sum_{b} f(b)<1$ ensures convergence of the sum over $b$ in $W_{\text {stringy }}$.

The contribution $W_{\text {stringy }}$ vanishes in the limit $M_{s} \rightarrow \infty$ with $\Lambda$ fixed, which gives the theory studied by [14].

\subsection{Contributions of D-string instantons}

The curve $C_{0}$ is isolated. Hence a Euclidean D-string wrapping it is a reasonable candidate to generate interesting instanton effects [37]. ${ }^{8}$ In the next few paragraphs we explain why this contributes a certain form of zero. Other curves in the del Pezzo are not isolated, and therefore are not expected to contribute to the superpotential.

The charge of $\phi_{C_{0}}$ is $(1,2,-3) \times M$. This is also the number of strings stretching from a D1 on $C_{0}$ to branes $1,2,3$. This is true by the following calculation. Let

$$
I=i_{\star} j_{\star} \mathcal{O}_{C_{0}}
$$

( $j: C_{0} \rightarrow \mathbb{F}_{1}$ is the inclusion) be the lift of $C_{0}$ to $B$. Then

$$
\int_{B} \operatorname{ch}(I) \wedge \operatorname{ch}\left(i_{\star} \mathcal{L}_{\alpha}\right) \wedge \operatorname{Td}(B)=(1,1,-1)_{\alpha}
$$

counts this number of strings per unit rank. Again these strings account for the anomalous charge of the instanton factor.

But what operator can soak up this charge? We find a satisfying answer as follows. Consider a euclidean D-string wrapping $C_{0}$ in the presence of the quiver. There are massless fermionic strings in the following representations:

$\begin{array}{cccc} & \mathrm{U}(M) & \mathrm{U}(2 M) & \mathrm{U}(3 M) \\ \psi & 1 & 1 & 3 M \\ \eta & 1 & \overline{2 M} & 1 \\ \lambda & \bar{M} & 1 & 1 .\end{array}$

The $\psi$ is in the antifundamental because $\mathcal{L}_{3}$ is an antibrane.

The following object is gauge invariant:

$$
L=\psi(u \lambda+u \cdot L \eta)
$$

The dot denotes a sum over $\mathrm{U}(M)$ colors. It is again reasonable to expect that $L$ is generated as the action for these fermionic modes by disc amplitudes. There is an important question regarding the flavor structure of $L$. Since the instanton on $C_{0}$ preserves the $\mathrm{SU}(2)$

\footnotetext{
${ }^{8}$ In many D3/D7 models, there are no possible contributions from D-string instantons, since all modes of $C_{2}$ on the internal space are projected out. In our model, some of the surviving axions arise from $C_{2}$, which is why this possibility even arises.
} 
isometry of $\mathbb{F}_{1}$, the action $L$ will respect the flavor $\mathrm{SU}(2)_{F}$ preserved by $W_{\text {tree }}$. This means only $L_{1,2}$ appear.

The integral over these modes of the instanton equals

$$
A=\int d \psi d \eta d \lambda e^{L}=\epsilon^{f g} \epsilon_{a b} \epsilon_{A B C} u_{A}^{i}\left(u_{B}^{j} \cdot L_{f}^{a}\right)\left(u_{C}^{k} \cdot L_{g}^{b}\right) C_{i j k}^{\mathcal{X}} .
$$

Here $C_{i j k}^{\mathcal{X}}$ is a intertwiner that projects the product of three 2's onto the completely antisymmetric $\mathbf{4}, \mathcal{X}=1, \ldots, 4$. But for $M=1$ this is

$$
A=\alpha_{\mathcal{X}}^{a} V_{a} \tilde{B}^{\mathcal{X}}
$$

where $V$ was defined above and

$$
\tilde{B}^{\mathcal{X}} \equiv C_{i j k}^{\mathcal{X}} \epsilon_{A B C} u_{A}^{i} u_{B}^{j} u_{C}^{k}
$$

If the $u$ 's are treated as bosonic objects, this is zero. So, a gauge invariant superpotential is

$$
e^{-t_{C_{0}}} V_{a} \tilde{B}^{\mathcal{X}} \alpha_{\mathcal{X}}^{a}
$$

which is classically zero. For $M>1$, the contribution also vanishes by Bose statistics of u's.

\section{Vacuum structure: on the capture of the runaway quiver}

One of the motivations for this work was the question of whether the runaway of the $d P_{1}$ quiver gauge theory [14] is cured by embedding into string theory. Here we apply the results of the previous sections to address this in the compact model introduced in $\S 3.2 .^{9}$ The basic idea is that once the Kähler moduli are stabilized by the D3 instanton contributions, they can play the role of FI parameters for the $\mathrm{U}(1)_{B}$ gauge symmetries; the resulting D-term potentials, which are finite in the compact model, then lift the runaway directions in open-string field space.

The full superpotential for $\mathcal{N}=1, D=4$ supergravity coupled to the quiver gauge theory has the form

$$
W_{\text {total }}=W_{\text {flux }}+W_{\text {tree }}+W_{\text {non-pert }}+y^{a}\left(Z V^{a}-\frac{1}{2} X_{i b} X_{j c} \epsilon^{a b c} \epsilon^{i j}\right)
$$

where $y^{a}, a=1,2,3$ are Lagrange multipliers. The added constraints reflect the fact that combinations $Z, V^{a}, X_{i b}$ of basic quiver fields $Q, u^{i}, L^{a}$ are not independent

$$
Z=\operatorname{det}_{f j} Q^{f} u_{j}, \quad X_{i a}=Q u_{i} L_{a}, \quad V^{a}=\frac{1}{2} L_{b} L_{c} \epsilon^{a b c} .
$$

Here $W_{\text {flux }}$ is the contribution due to background fluxes which is responsible for stabilization of complex structure moduli. $W_{\text {tree }}$ is the tree level superpotential in quiver gauge

\footnotetext{
${ }^{9}$ In the discussion below we ignore the global issue of stabilization of moduli of extra D3-branes which are required to saturate the $C^{(4)}$ tadpole constraint.
} 
theory. Finally, $W_{\text {non-pert }}$ includes D3-instanton corrections $W_{\text {inst }}=W_{\mathrm{ADS}}+W_{\text {stringy }}$ computed in this paper as well as $W_{\text {gaugino, }}$, the contribution from gaugino condensation in pure $\mathrm{SO}(8)$ gauge theory supported on the divisor at infinity $D_{6} \cdot{ }^{10}$ This divisor is a fixed set under orientifold action and therefore an O7-plane is wrapped on it together with 4 D7-branes. Since $D_{6}$ does not intersect $D_{5}$, where the quiver branes are wrapped, this particular contribution does not contain operators from the quiver gauge theory, and is just given by ${ }^{11}$

$$
W_{\text {gaugino }} \sim \Lambda_{\mathrm{SO}(8)}^{3} e^{-\alpha S_{3}},
$$

where $\alpha$ is a number less than one. Here

$$
S_{3}=\int_{B}\left(\frac{1}{2 \pi g_{s}} J^{2}+i C^{(4)}\right) D_{6} .
$$

Parametrizing the Kähler form as in previous sections: $J=r_{5} D_{5}+r_{2} D_{2}+r_{3} D_{3}$ gives

$$
\operatorname{Re}\left(S_{3}\right)=r_{2} r_{3}-\frac{1}{2} r_{3}^{2}
$$

Collecting all the non-perturbative corrections we find

$$
W_{\text {non-pert }}=\Lambda^{7}\left(\frac{1}{Z} e^{-S_{1}}+\frac{1}{M_{s}^{6}} \sum_{b>1 \& b \leq-1} V_{3} f(b) e^{-S_{1}+(b-1) S_{2}}\right)+\Lambda_{\mathrm{SO}(8)}^{3} e^{-\alpha S_{3}} .
$$

Let us denote $\rho_{a}=\operatorname{Re}\left(S_{a}\right)$. The regime of validity of (6.3) is

$$
\rho_{3} \gg \rho_{1} \gg 1, \quad\left|S_{2}\right| \ll 1 .
$$

The conditions on $S_{1}$ and $S_{3}$ are imposed to justify neglecting higher exponentials of the type $e^{-n S_{1}}$ for $n>1$. Please note that we are forced to require $\rho_{3} \gg \rho_{1}$ since this is the only way to have $\rho_{3} \gg 1, \rho_{1} \gg 1$ and, at the same time, ensure that sizes of the 2-cycles $C_{0}, f$ as well as of the $\mathbb{P}^{1}$-fiber are non-negative:

$$
r_{2} \geq r_{3}+r_{5}, \quad r_{3} \geq 2 r_{5}, \quad r_{5} \geq 0 .
$$

Meanwhile, the condition on $S_{2}$ is the analytic continuation of the condition for the alignment of the three quiver nodes discussed in $\S 5.2$.

The fields $Z$ and $X_{i a}$ can be integrated out in the same manner as in [14] and the effective superpotential takes the form

$$
W_{\text {eff }}=W_{\text {flux }}+3\left(h^{2} \Lambda^{7} V_{3} e^{-S_{1}}\right)^{1 / 3}+\frac{\Lambda^{7}}{M_{s}^{6}} \sum_{b>1 \& b \leq-1} V_{3} f(b) e^{-S_{1}+(b-1) S_{2}}+\Lambda_{\mathrm{SO}(8)}^{3} e^{-\alpha S_{3}} .
$$

Note that there is no susy vacuum in the regime of validity (6.4) of $W_{\text {eff }}$ since, for example, the equations $D_{S_{2}} W_{\text {eff }}=0$ and $D_{V_{3}} W_{\text {eff }}=0$ are incompatible with each other. Indeed, in the regime (6.4),

$$
\kappa^{2} \partial_{V_{3}} K \sim \frac{\rho_{1}}{\rho_{3}} \ll 1, \quad \kappa^{2} \partial_{S_{2}} K \sim \frac{1}{\sqrt{\rho_{3}}} \ll 1 .
$$

\footnotetext{
${ }^{10}$ This is model dependent, and assumes we did not choose to put a nontrivial vector bundle in this $\mathrm{SO}(8)$ to absorb some of the D3 charge tadpole.

${ }^{11}$ We ignore possible dependence on complex structure moduli.
} 
Therefore, $D_{S_{2}} W_{\text {eff }} \sim \partial_{S_{2}} W_{\text {eff }}$ and $D_{V_{3}} W_{\text {eff }} \sim \partial_{V_{3}} W_{\text {eff }}$ and the equations $\partial_{S_{2}} W_{\text {eff }}=0$ and $\partial_{V_{3}} W_{\text {eff }}=0$ are incompatible with each other.

To find out if there is a stable minimum one should have a closer look at the full scalar potential. Besides the superpotential $W_{\text {eff }}$ we need the Kähler potential. We would like to emphasize that the regime of validity (6.4) implies

$$
r_{2} \sim(3 / 2) r_{3} \gg r_{5} \gg 1
$$

which is consistent with taking the large radius limit. So one may use for the Kähler potential the standard large radius expression:

$$
\kappa^{2} K=-2 \log \left(\operatorname{vol}_{B}+\frac{\operatorname{vol}_{D_{5}}}{M_{p l}^{2}} \sqrt{V_{a} V_{a}^{*}}\right), \quad \kappa^{2}=\frac{1}{M_{p l}^{2}} .
$$

Here $\operatorname{vol}_{B}$ is the volume of the threefold $B$ in string units,

$$
\operatorname{vol}_{B}=\frac{1}{3 !} \int_{B} J^{3}=\frac{1}{3 !}\left(r_{5}^{3}-3 r_{3} r_{5}^{2}-3 r_{5} r_{3}^{2}-6 r_{2} r_{5}^{2}+6 r_{2} r_{3} r_{5}\right)
$$

and $\operatorname{vol}_{D_{5}}$ is the dimensionless volume of the divisor $D_{5}$

$$
\operatorname{vol}_{D_{5}}=\frac{1}{2}\left(8 r_{5}^{2}-r_{3}^{2}-2 r_{3} r_{5}-4 r_{2} r_{5}+2 r_{2} r_{3}\right)
$$

where $r_{2}, r_{3}, r_{5}$ are expressed in terms of

$$
\rho_{a}=\operatorname{Re}\left(S_{a}\right), \quad a=1,2,3
$$

by using the definitions of $S_{a}$ given in (5.4), (5.9) and (6.2). In writing (6.7) we have used the kinetic term ${ }^{12}$ for $V_{a}$

$$
\int_{M^{4}} \sqrt{g} \frac{\operatorname{vol}_{D_{5}}}{\operatorname{vol}_{B}} g^{\mu \nu} \partial_{\mu} V_{a} \partial_{\nu} V_{a}^{*}
$$

Equipped with the superpotential $W_{\text {eff }}$ and the Kähler potential $K$, one may use the standard formula for the scalar potential in $\mathcal{N}=1, D=4$ supergravity:

$$
V=\exp \left(\kappa^{2} K\right)\left(K^{I \bar{J}} W_{e f f ; I} W_{e f f ; \bar{J}}^{*}-3 \kappa^{2} W_{\mathrm{eff}}^{*} W_{\mathrm{eff}}\right)+\frac{1}{2 g_{X}^{2}} \sum_{a=1}^{3}\left(D_{a}\right)^{2}
$$

where the $\mathrm{U}(1)$ D-terms are given by:

$$
D_{1}=-D_{2}=-2\left(V^{a} \partial_{V^{a}} K+\partial_{\rho_{1}} K\right), \quad D_{3}=0 .
$$

We have used the fact that both $V^{a}$ and $e^{S_{1}}$ have $\mathrm{U}(1)$ charges $(-2,2,0)$ and $e^{S_{2}}$ and $e^{S_{3}}$ are neutral.

It is a hard technical problem to analyze the dynamics of the six complex scalars $V_{1}, V_{2}, V_{3}, S_{1}, S_{2}, S_{3}$ which enter in (6.8). However, we now propose a strategy to show that

\footnotetext{
${ }^{12}$ Recall that $d s_{10}^{2}=\operatorname{vol}_{B}^{-1} d s_{4}^{2}+d s_{6}^{2}$.
} 
a minimum of (6.8) does exist. If $W_{\text {flux }}$ is large, one may write $W_{\text {eff }}=W_{\text {flux }}+W_{\text {correction }}$ where

$$
W_{\text {correction }}=3\left(h^{2} \Lambda^{7} V_{3} e^{-S_{1}}\right)^{1 / 3}+\frac{\Lambda^{7}}{M_{s}^{6}} \sum_{b>1 \& b \leq-1} V_{3} f(b) e^{-S_{1}+(b-1) S_{2}}+\Lambda_{\mathrm{SO}(8)}^{3} e^{-\alpha S_{3}} .
$$

Since $W_{\text {flux }}$ is large it is reasonable to keep only terms where $W_{\text {correction }}$ appears no more than once. Also, for $\operatorname{vol}_{B}$ large but finite, by adjusting $W_{\text {flux }}$ one can make $\frac{1}{\operatorname{vol}_{B}}\left|W_{\text {flux }}\right|^{2}$ much larger than the D-terms and discard the latter. In this regime one may approximate $V_{\text {eff }}$ as follows

$$
\frac{1}{\operatorname{vol}_{B}}\left[\left(-3 \kappa^{2}+K^{I \bar{J}} \partial_{I}\left(\kappa^{2} K\right) \partial_{\bar{J}}\left(\kappa^{2} K\right)\right)\left|W_{\text {flux }}\right|^{2}+K^{I \bar{J}}\left[\partial_{I}\left(\kappa^{2} K\right) W_{\text {flux }} \partial_{\bar{J}}\left(\bar{W}_{\text {correction }}\right)+c c\right]\right] \text {. }
$$

Now, in order to argue for the existence of a minimum of the theory, it is sufficient to check two things:

(i) Show that dynamics of axions $\operatorname{Im} S_{a}$ and phases $\arg V_{a}$ are such that they stabilize (for fixed values of $\operatorname{Re} S_{a}$ and $\left|V_{a}\right|$ ) at values for which mixed terms in $V_{\text {eff }}$, which involve $W_{\text {flux }}$ and $W_{\text {correction }}$, are negative. ${ }^{13}$

(ii) Check that $-3 \kappa^{2}+K^{I \bar{J}} \partial_{I}\left(\kappa^{2} K\right) \partial_{\bar{J}}\left(\kappa^{2} K\right) \leq 0$ in the regime of validity (6.6) of $W_{\text {eff }}$.

If both conditions are achieved, the potential is negative at some configuration inside the regime of validity. Recall now that in the limit $\operatorname{vol}_{B}$, $\operatorname{vol}_{D_{5}} \rightarrow \infty$ (also in the regime of validity) $V_{\text {eff }} \rightarrow 0$. Since $V_{\text {eff }}$ is bounded below (even outside our regime of control, as long as the full model is well-defined), there is a minimum somewhere, though perhaps outside of the regime of validity of $V_{\text {eff }}$. It would be interesting to explore the conditions under which one can find minima with exponentially small supersymmetry breaking due to the dynamics of the quiver gauge theory.

Also, if $\left|V_{3}\right|$ is large enough

$$
\frac{\kappa^{2}\left|W_{\text {flux }}\right|^{2}}{\operatorname{vol}_{B}} \ll \frac{\left|V_{3}\right|^{2} \operatorname{vol}_{D_{5}}^{2}}{\operatorname{vol}_{B}^{2}},
$$

the D-terms dominate over the F-term and the potential at infinity $\operatorname{vol}_{B}, \operatorname{vol}_{D_{5}} \rightarrow \infty$ is positive (and approaching zero). Combined with $V_{\text {eff }}<0$ at some configuration inside the regime of validity this suggests existence of a dS maximum in the regime of validity of $V_{\text {eff }}$.

\section{A. Mixed anomalies}

There is mixed anomaly $\mathrm{U}(1)_{R} \mathrm{U}(1)_{a} \mathrm{U}(1)_{b}$ in the theory with baryonic $\mathrm{U}(1)_{B}$ factors included:

$$
\partial_{\mu} j_{R}^{\mu}=-2 \lambda_{R} r_{a} r_{b} \star\left(F_{a} \wedge F_{b}\right) .
$$

\footnotetext{
${ }^{13}$ The sign of these mixed terms depends on axions and phases.
} 
Here $a, b=1,2,3$ label the nodes of the quiver and the gauge field strength $F_{a}$ of the $\mathrm{U}(1)_{a}$ factor is a 2 -form in $\mathbb{R}^{4}$.

The compact axions $\phi_{\mathbb{F}_{1}}, \phi_{C_{0}}, \phi_{f}$ should not transform under R-symmetry since they couple to quiver gauge theory as:

$$
I_{\text {comp. }}=\operatorname{tr}_{1} F^{2} \phi_{\mathbb{F}_{1}}+\operatorname{tr}_{2} F^{2}\left(\phi_{\mathbb{F}_{1}}+\phi_{f}+\phi_{C_{0}}\right)-\operatorname{tr}_{3} F^{2}\left(\phi_{\mathbb{F}_{1}}+\phi_{f}\right) .
$$

Any shift of $\phi_{\mathbb{F}_{1}}, \phi_{C_{0}}, \phi_{f}$ would result in generating another anomaly $\mathrm{U}(1)_{R} \mathrm{SU}(N)^{2}$ which is otherwise absent in the theory.

We propose a mechanism for cancellation of an $\mathrm{U}(1)_{R} \mathrm{U}(1)_{a} \mathrm{U}(1)_{b}$ anomaly which involves $\hat{C}_{2}$, the $\mathrm{RR} 2$-form in $\mathbb{R}^{4}$ and $C_{2}^{\prime}$, the 2 -form in $\mathbb{R}^{4}$ obtained from KK reduction of the RR 4-form:

$$
C_{4}=C_{2}^{\prime} \wedge \omega_{f}+\ldots
$$

The specific KK component $C_{2}^{\prime}$ is chosen due to the property that it couples only to $F_{2}$.

These 2 -forms couple to the gauge fields as follows

$$
I_{\text {non-comp }}=\frac{1}{12} \sum_{a=1}^{3} r_{a} \int_{M^{4}} \hat{C}_{2} \wedge F_{a}+\frac{1}{2} \int_{M^{4}} \hat{C}_{2} \wedge F_{2}+2 \int_{M^{4}} C_{2}^{\prime} \wedge F_{2} .
$$

Now we require the following non-trivial transformations under R-symmetry:

$$
\delta_{R} \hat{C}_{2}=24 \lambda_{R} \sum_{a=1}^{3} r_{a} F_{a}, \quad \delta_{R} C_{2}^{\prime}=-6 \lambda_{R} \sum_{a=1}^{3} r_{a} F_{a}
$$

As a result of these transformations

$$
\delta_{R} I_{\text {non-comp }}=2 \lambda_{R} r_{a} r_{b} F_{a} \wedge F_{b}
$$

and the anomaly A.1. is completely cancelled.

\section{Acknowledgments}

We thank Riccardo Argurio, Matteo Bertolini, Chris Herzog, Ken Intriligator, Anton Kapustin, Igor Klebanov, Melissa Liu, Liam McAllister, Hirosi Ooguri, Eva Silverstein, Peter Svrcek, Jay Wacker, Johannes Walcher and Martijn Wijnholt for helpful discussions. The work of the Stanford authors was supported in part by the NSF under grant PHY-0244728, the DOE under contract DE-AC03-76SF00515, and a David and Lucile Packard Foundation Fellowship for Science and Engineering. The work of N.S. was supported in part by NSF grants PHY-0244821 and DMS-0244464. The work of J.M. is supported in part by funds provided by the U.S. Department of Energy (D.O.E.) under cooperative research agreement DE-FG0205ER41360. S.K. is grateful to the KITP for hospitality. 


\section{References}

[1] E. Witten, Dynamical breaking of supersymmetry, Nucl. Phys. B 188 (1981) 513.

[2] E. Witten, Small instantons in string theory, Nucl. Phys. B 460 (1996) 541 hep-th/9511030;

M.R. Douglas, Branes within branes, hep-th/9512077.

[3] E. Witten, Non-perturbative superpotentials in string theory, Nucl. Phys. B 474 (1996) 343 hep-th/9604030.

[4] S. Kachru, R. Kallosh, A. Linde and S.P. Trivedi, De Sitter vacua in string theory, Phys. Rev. D 68 (2003) 046005 hep-th/0301240.

[5] F. Denef, M.R. Douglas and B. Florea, Building a better racetrack, JHEP 06 (2004) 034 hep-th/0404257;

F. Denef, M.R. Douglas, B. Florea, A. Grassi and S. Kachru, Fixing all moduli in a simple

F-theory compactification, Adv. Theor. Math. Phys. 9 (2005) 861 hep-th/0503124;

D. Lüst, S. Reffert, W. Schulgin and S. Stieberger, Moduli stabilization in type-IIB orientifolds. I: orbifold limits, Nucl. Phys. B 766 (2007) 68 hep-th/0506090;

D. Lüst, S. Reffert, E. Scheidegger, W. Schulgin and S. Stieberger, Moduli stabilization in type-IIB orientifolds. II, Nucl. Phys. B 766 (2007) 178 hep-th/0609013;

[6] V. Balasubramanian, P. Berglund, J.P. Conlon and F. Quevedo, Systematics of moduli stabilisation in Calabi-Yau flux compactifications, JHEP 03 (2005) 007 hep-th/0502058.

[7] O.J. Ganor, A note on zeroes of superpotentials in F-theory, Nucl. Phys. B 499 (1997) 55 hep-th/9612077.

[8] M. Dine, N. Seiberg and E. Witten, Fayet-iliopoulos terms in string theory, Nucl. Phys. B 289 (1987) 589.

[9] M.R. Douglas and G.W. Moore, D-branes, quivers and ALE instantons, hep-th/9603167.

[10] L.E. Ibáñez, R. Rabadán and A.M. Uranga, Anomalous U(1)'s in type-I and type-IIB D =4, $N=1$ string vacua, Nucl. Phys. B 542 (1999) 112 hep-th/9808139.

[11] D. Berenstein, C.P. Herzog, P. Ouyang and S. Pinansky, Supersymmetry breaking from a Calabi-Yau singularity, JHEP 09 (2005) 084 hep-th/0505029.

[12] S. Franco, A. Hanany, F. Saad and A.M. Uranga, Fractional branes and dynamical supersymmetry breaking, JHEP 01 (2006) 011 hep-th/0505040.

[13] M. Bertolini, F. Bigazzi and A.L. Cotrone, Supersymmetry breaking at the end of a cascade of Seiberg dualities, Phys. Rev. D 72 (2005) 061902 hep-th/0505055.

[14] K. Intriligator and N. Seiberg, The runaway quiver, JHEP 02 (2006) 031 hep-th/0512347.

[15] R. Argurio, M. Bertolini, C. Closset and S. Cremonesi, On stable non-supersymmetric vacua at the bottom of cascading theories, JHEP 09 (2006) 030 hep-th/0606175.

[16] D.-E. Diaconescu, B. Florea, S. Kachru and P. Svrcek, Gauge-mediated supersymmetry breaking in string compactifications, JHEP 02 (2006) 020 hep-th/0512170.

[17] I. Affleck, M. Dine and N. Seiberg, Dynamical supersymmetry breaking in supersymmetric QCD, Nucl. Phys. B 241 (1984) 493.

[18] H. Verlinde and M. Wijnholt, Building the standard model on a D3-brane, JHEP 01 (2007) 106 hep-th/0508089. 
[19] M. Buican, D. Malyshev, D.R. Morrison, M. Wijnholt and H. Verlinde, D-branes at singularities, compactification and hypercharge, JHEP 01 (2007) 107 hep-th/0610007.

[20] L.E. Ibáñez and A.M. Uranga, Neutrino majorana masses from string theory instanton effects, JHEP 03 (2007) 052 hep-th/0609213].

[21] R. Blumenhagen, M. Cvetič and T. Weigand, Spacetime instanton corrections in $4 D$ string vacua - the seesaw mechanism for D-brane models, Nucl. Phys. B 771 (2007) 113 hep-th/0609191.

[22] M. Haack, D. Krefl, D. Lüst, A. Van Proeyen and M. Zagermann, Gaugino condensates and D-terms from D7-branes, JHEP 01 (2007) 078 hep-th/0609211.

[23] J. Preskill, Gauge anomalies in an effective field theory, Ann. Phys. (NY) 210 (1991) 323.

[24] M. Berkooz, M.R. Douglas and R.G. Leigh, Branes intersecting at angles, Nucl. Phys. B 480 (1996) 265 hep-th/9606139.

[25] N. Arkani-Hamed, M. Dine and S.P. Martin, Dynamical supersymmetry breaking in models with a Green-Schwarz mechanism, Phys. Lett. B 431 (1998) 329 hep-ph/9803432.

[26] S. Kachru, M.B. Schulz and S. Trivedi, Moduli stabilization from fluxes in a simple IIB orientifold, JHEP 10 (2003) 007 hep-th/0201028.

[27] E. Witten, Phases of $N=2$ theories in two dimensions, Nucl. Phys. B 403 (1993) 159 hep-th/9301042.

[28] R. Minasian and G.W. Moore, $k$-theory and Ramond-Ramond charge, JHEP 11 (1997) 002 hep-th/9710230.

[29] J. Polchinski, String theory, vol.2, Cambridge University Press (1998).

[30] W. Fulton, Introduction to toric varieties, Princeton University Press (1993).

[31] L. Martucci, J. Rosseel, D. Van den Bleeken and A. Van Proeyen, Dirac actions for D-branes on backgrounds with fluxes, Class. and Quant. Grav. 22 (2005) 2745 hep-th/0504041.

[32] N. Saulina, Topological constraints on stabilized flux vacua, Nucl. Phys. B 720 (2005) 203 hep-th/0503125.

[33] R. Kallosh, A.-K. Kashani-Poor and A. Tomasiello, Counting fermionic zero modes on M5 with fluxes, JHEP 06 (2005) 069 hep-th/0503138.

[34] E. Bergshoeff, R. Kallosh, A.-K. Kashani-Poor, D. Sorokin and A. Tomasiello, An index for the Dirac operator on D3 branes with background fluxes, JHEP 10 (2005) 102 hep-th/0507069.

[35] M. Bershadsky, A. Johansen, T. Pantev, V. Sadov and C. Vafa, F-theory, geometric engineering and $N=1$ dualities, Nucl. Phys. B 505 (1997) 153 hep-th/9612052.

[36] Talk given by J. McGreevy at Strings 2006, Beijing, On the capture of runaway quivers, online at http://strings06.itp.ac.cn/talk-files/mcgreevy.pd.

[37] E. Witten, World-sheet corrections via D-instantons, JHEP 02 (2000) 030 hep-th/9907041. 